\title{
The Determination of Feasible Control Variables for Geoengineering and Weather Modification Based on the Theory of Sensitivity in Dynamical Systems
}

\author{
Sergei A. Soldatenko and Rafael M. Yusupov \\ St. Petersburg Institute for Informatics and Automation, The Russian Academy of Sciences, No. 39, 14th Line, \\ St. Petersburg 199178, Russia
}

Correspondence should be addressed to Sergei A. Soldatenko; soldatenko@iias.spb.su

Received 25 November 2015; Revised 2 May 2016; Accepted 23 May 2016

Academic Editor: Ai-Guo Wu

Copyright (C) 2016 S. A. Soldatenko and R. M. Yusupov. This is an open access article distributed under the Creative Commons Attribution License, which permits unrestricted use, distribution, and reproduction in any medium, provided the original work is properly cited.

Geophysical cybernetics allows for exploring weather and climate modification (geoengineering) as an optimal control problem in which the Earth's climate system is considered as a control system and the role of controller is given to human operators. In mathematical models used in climate studies control actions that manipulate the weather and climate can be expressed via variations in model parameters that act as controls. In this paper, we propose the "instability-sensitivity" approach that allows for determining feasible control variables in geoengineering. The method is based on the sensitivity analysis of mathematical models that describe various types of natural instability phenomena. The applicability of this technique is illustrated by a model of atmospheric baroclinic instability since this physical mechanism plays a significant role in the general circulation of the atmosphere and, consequently, in climate formation. The growth rate of baroclinic unstable waves is taken as an indicator of control manipulations. The information obtained via calculated sensitivity coefficients is very beneficial for assessing the physical feasibility of methods of control of the large-scale atmospheric dynamics and for designing optimal control systems for climatic processes. It also provides insight into potential future changes in baroclinic waves, as a result of a changing climate.

\section{Introduction}

Weather modification is the operation of deliberately altering the atmosphere that leads to changes in the natural evolution of physical and dynamical atmospheric processes. Predominantly, weather modification is successfully accomplished via cloud seeding in order to affect precipitation for the purpose of the local water supply (e.g., [1-4]). Many countries currently practice cloud seeding operationally. The next successful example of weather modification is fog dispersal to improve visibility at airports by heating or seeding [5]. Some attempts taken in different countries to reduce damage from hazardous weather events such as hurricanes, strong tornado and thunderstorm winds, hail, lighting, and floods, unfortunately, have not been so successful. The detailed retrospective review and the current status of weather modification with the findings and relevant concepts were considered, for example, in [6-8]. Over the last decades, the weather modification has transitioned from local scale operations to a global weather modification known also as geoengineering. Geoengineering, or climate engineering, is a deliberate and purposeful large-scale modification of the Earth's climate system (ECS) and, first of all, the atmosphere, which is the most unstable and fast-changing element of the ECS [9]. Geoengineering was offered by scientific community as a response to global warming, which is happening. Since mankind is causing global warming by anthropogenic $\mathrm{CO}_{2}$ emissions [10], the most obvious idea to reduce the consequences of global climate change is a sequestration of anthropogenic greenhouse gas (GHG) emissions. However, this is unlikely achievable in foreseeable future due to the continuing growth of the world economy and population. Scientists and engineers proposed several solutions to stabilize the global climate (e.g., [11, 12]). These solutions can be divided into two main categories: carbon dioxide removal technologies (CDR) and solar radiation management (SRM). CDR 
technologies include, for example, various engineered solutions that remove GHGs directly from the atmosphere, using big machines or chemical absorbents. SRM techniques aim mainly to reflect some percentage of the solar radiation back into the outer space. This can be achieved by changing the planetary albedo (increasing the reflectiveness of the Earth's surface or clouds), dispersing small particles into the stratosphere, or deploying mirrors in the upper atmosphere to reflect sunlight. However, all of these technologies introduce uncertainties and unexpected consequences that must be explored.

Let both weather modification and climate engineering hereafter be referred to as the geoengineering. Realization of geoengineering projects is a purposeful process, that is, the process imminently connected with a specific objective that can be formulated in various ways. In this context geoengineering is per se the process of controlling the ECS. However, geoengineering is still considered outside of the scope of control theory as an intentional action to influence natural processes. Meanwhile in the late 1970s a uniform methodology for control geophysical processes, including processes occurring in the ECS, was formulated on the basis of the ideas from cybernetics by one of the authors of this paper [13]. In this monograph, the concept of geophysical cybernetics was introduced as the new research area within the control theory. Geophysical cybernetics explores a self-regulating feedback cybernetic system, in which the ECS is considered as the control object and the role of the controller is given to human operators. From the standpoint of geophysical cybernetics, climate and weather manipulation represents an optimal control problem, which aims to synthesize the control law that ensures the achievement of the desired results that can be expressed in terms of extremal problem [13, 14]. In our previous publications [14-16] an optimal control problem for the ECS has been conceptually formulated in both probabilistic and deterministic manner. Let us emphasize that the ECS is a unique natural physical system with a large number of specific attributes [17], which makes the control problem for this system extremely complex. The development of physically feasible methods to control the ECS requires the determination of feasible control mechanisms and variables. The theory of sensitivity in dynamical systems $[18,19]$ serves as a theoretical instrument for solving this problem.

This paper presents a short description of the ECS as a control system, emphasizing its unique physical properties relevant to the control problems of large dynamical systems. It is also noted that the amount of energy released during processes that drive the ECS is orders of magnitudes greater than human capabilities, which makes the control of the ECS via direct interventions very problematic. However, some physical and dynamical processes occurring in the ECS are inherently unstable, which apparently allows one to implement geoengineering projects using significantly less energy resources in comparison with the energy of natural processes. Thus, the study of various types of instabilities in ECS is very important for developing an appropriate control strategy. The instability of natural physical processes is mathematically studied as a problem of finding the necessary conditions for the growth of the infinitesimal perturbations. Since these conditions are expressed in terms of some model parameters, by using sensitivity analysis, we can, first, determine parameters that can be considered as controls and, second, make a conclusion on the hypothetical possibility of control of physical process under consideration. To illustrate this "instability-sensitivity" approach we, as an example, consider the atmospheric baroclinic instability as the controlled object since this type of hydrodynamic instability plays a substantial role in the formation of large-scale atmospheric eddies in the extratropical atmosphere and, therefore, in the general circulations of the atmosphere and climate [20]. To explore the response of baroclinic instability to geoengineering interventions a multilayer geostrophic model of the atmosphere is applied. The growth rate of baroclinic unstable waves is taken as an indicator of control manipulations. The information obtained via calculated sensitivity coefficients is very beneficial for assessing the physical feasibility of methods of control of the large-scale atmospheric dynamics and for designing optimal control systems for climatic processes.

\section{Climate System as a Unique Control System}

The ECS is a unique and peculiar natural physical system that is extremely difficult to control since it possesses a number of specific properties, including but not limited to the following $[14,17,21,22]$.

(i) The ECS is a complex, interactive system with a wide variety of positive and negative feedback mechanisms. The ECS consists of the atmosphere, ocean, sea-ice, land surface, and other bodies of water and includes global carbon cycle, chemistry, and aerosols. These natural subsystems have substantial differences in their physical and chemical properties, structure, and behavior; they can be strongly or weakly coupled and linked together by means of coupling physical mechanisms.

(ii) Dynamical and physical processes in the ECS occur over a broad spectrum of scales in both space and time. Time scales are varied from seconds (turbulent fluctuations) to dozens of years (climate change and variability). Since the ECS is a global system its spatial spectrum of motions covers molecular to planetary scales. It is important that dynamical processes in the atmosphere and ocean are nonlinear and chaotic

(iii) Processes in the ECS oscillate due to both internal factors (natural oscillations) and external forcing (forced oscillations). Natural oscillations are due to the internal instability of ECS with respect to stochastic infinitesimal disturbances. Human impact on the ECS, both intentional and unintentional, is considered as external forcing.

The ECS has certainly a number of other specific attributes that make it a unique and complex physical system. One of the most effective instruments in studying the ECS is mathematical/numerical modeling. Climate mathematical models used in a variety of applications are commonly deterministic and derived from a set of multidimensional 
nonlinear differential equations in partial derivatives, which are the equations of fluid dynamics and thermodynamics. Models also take into consideration the specific properties of ECS as well as its cycles such as water, nitrogen, oxygen, and carbon cycles.

An optimal control problem for the ECS remains poorly studied due to its relative novelty and enormous complexity $[15,16]$. To develop a general framework for optimal control of the ECS, the following should be taken into consideration.

(i) The ECS is a spatially distributed system, therefore the control actions for manipulating this system should also be distributed in space. However, implementation of such controls is weakly developed.

(ii) Processes in the ECS possess enormous energy potential. It is hardly possible to provide control actions whose energy is comparable to the energy of natural physical processes. Therefore, the identification of sensitive points, in which the ECS is in an unstable state, is a critical issue.

(iii) Large-scale and huge energy of climate-driven processes impose very strict requirements for the accuracy and reliability of control systems, since even minor errors in control actions can be disastrous.

(iv) Processes in the ECS are interconnected; therefore changes in the dynamics of some processes can result in uncontrollable consequences.

(v) Control actions to perform geoengineering and weather modification operations must be physically feasible and executable.

Consequently, discussing geoengineering within the scope of optimal control theory, we are faced with a number of problems including the problem of choosing variables that can be considered as controls. Mathematical climate models incorporate a certain number of physical processes responsible for the transformation of energy. These processes represent natural control mechanisms of the ECS that can be considered as potential artificial control mechanisms. Unfortunately, these physical mechanisms cannot be explicitly identified and represented in climate models due to models' discrete spatial-temporal structure and can only be described parametrically under some simplified assumptions. Some of the newly introduced parameters together with parameters that describe the external forcing can be considered as control variables (parameters). Then geoengineering actions can mathematically be represented via variations in parameters that act as controls. By using sensitivity analysis, we can explore the influence of variations in control parameters on the behavior of the ECS and, therefore, evaluate the hypothetical possibility of various physical mechanisms to control the ECS. However, we need to keep in mind that control methods should be, on the one hand, physically feasible and, on the other hand, technically executable. Many physical processes occurring in the ECS are inherently unstable $[20,23]$. This gives the possibility to control the ECS via natural instability mechanisms. Apparently, the atmosphere, which is the most rapidly moving and changing components of the ECS, represents the most suitable system to be controlled.

\section{The Model of Baroclinic Instability in the Atmosphere}

Let us consider the set of the so-called primitive equations in isobaric coordinate system commonly used in modelling large-scale atmospheric flows [23]:

$$
\begin{aligned}
\frac{\partial u}{\partial t}+u \frac{\partial u}{\partial x}+v \frac{\partial u}{\partial y}+\omega \frac{\partial u}{\partial p}-f v & =-\frac{\partial \Phi}{\partial x}, \\
\frac{\partial v}{\partial t}+u \frac{\partial v}{\partial x}+v \frac{\partial v}{\partial y}+\omega \frac{\partial v}{\partial p}+f u & =-\frac{\partial \Phi}{\partial y}, \\
\frac{R T}{p} & =-\frac{\partial \Phi}{\partial p}, \\
\frac{\partial u}{\partial x}+\frac{\partial v}{\partial y}+\frac{\partial \omega}{\partial p} & =0, \\
\frac{\partial T}{\partial t}+u \frac{\partial T}{\partial x}+v \frac{\partial T}{\partial y}-\frac{R T}{g p}\left(\gamma_{d}-\gamma\right) \omega & =0 .
\end{aligned}
$$

Here $u$ and $v$ are the horizontal velocity components in the directions $x$ and $y$, respectively (the horizontal coordinates, $x$ and $y$, are directed eastward and northward); $\omega \equiv d p / d t$ is pressure vertical velocity, where $p$ is pressure; $\Phi$ is the geopotential; $T$ is the temperature; $R$ is the gas constant for dry air; $f$ is the Coriolis parameter; $g$ is the gravity acceleration; $\gamma_{d}$ is the dry adiabatic lapse rate; $\gamma$ is the reference state lapse rate. We will employ the $\beta$-plane approximation so that the Coriolis parameter $f$ is represented as $f=f_{0}+\beta y$, where $f_{0}$ is a standard value of the Coriolis parameter at the midlatitude and $\beta=\partial f / \partial y$ is the latitudinal gradient of $f$.

Let us make some comments regarding the set of equations (1)-(5). These are nonlinear differential equations that are used to describe adiabatic large-scale atmospheric dynamics. Equations (1) and (2) are the momentum equations, which mathematically express Newton's second law of motion. Equation (3) is a hydrostatic equation. The continuity equation (4) expresses the conservation of mass and (5) is a thermal energy equation representing the first law of thermodynamics.

Baroclinic instability is commonly explored by linearizing the model equations around some unperturbed reference (basic) flow and then solution of the problem can be found using initial-value or eigenvalue approaches. We suppose that the atmospheric reference flow, defined by $\bar{u}, \bar{v}, \bar{\omega}, \bar{T}, \bar{\Phi}$, is geostrophic (i.e., the Coriolis force and pressure gradient forces are in balance) and satisfies the following relations:

$$
\begin{aligned}
\bar{u} & =-\frac{1}{f_{0}} \frac{\partial \bar{\Phi}}{\partial y}, \\
\bar{v} & =0, \\
\bar{\omega} & =0, \\
\frac{\partial \bar{\Phi}}{\partial p} & =-\frac{R \bar{T}}{p},
\end{aligned}
$$




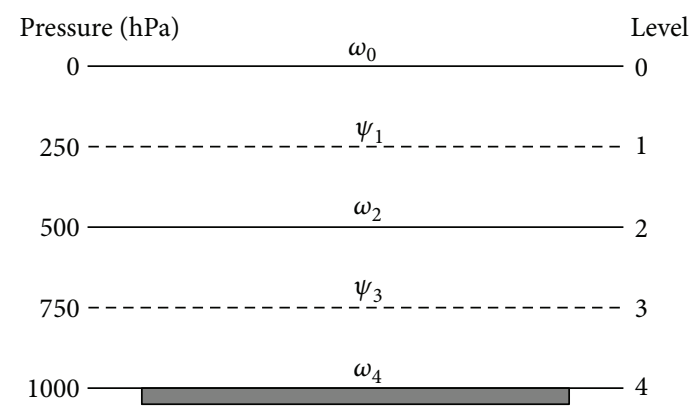

FIGURE 1: The distribution of levels and arrangement of variables in the vertical for two-layer model.

where $\bar{T}=\bar{T}(y, p)$. The reference state (6) is a solution of (1)-(5) that describes the zonal flow

$$
\frac{\partial \bar{u}}{\partial p}=\frac{R}{f_{0} p} \frac{\partial \bar{T}}{\partial y},
$$

which matches the specified distribution of the zonally averaged temperature $\bar{T}(y, p)$ and represents thermal wind balance. Let us underline that the geostrophic approximation used in this study is valid to high accuracy for the large-scale atmospheric flows. Then, with geostrophic assumption, (1)(5) reduced to the vorticity equation and the thermodynamic equation [23]:

$$
\begin{gathered}
\left(\frac{\partial}{\partial t}+u \frac{\partial}{\partial x}+v \frac{\partial}{\partial y}\right) \nabla^{2} \psi+\beta \frac{\partial \psi}{\partial p}=f_{0} \frac{\partial \omega}{\partial p} \\
\left(\frac{\partial}{\partial t}+u \frac{\partial}{\partial x}+v \frac{\partial}{\partial y}\right) \frac{\partial \psi}{\partial p}+\frac{\sigma}{f_{0}} \omega=0 .
\end{gathered}
$$

Here $\psi=\Phi / f_{0}$ is a geostrophic stream function and $\sigma=$ $\left(R^{2} T / g p^{2}\right)\left(\gamma_{d}-\gamma\right)$ is the static stability parameter, where $g$ is the gravity acceleration. To conserve the mass, the following boundary conditions are used for the pressure velocity [23]:

$$
\omega=0 \quad \text { at } p=0, p=P_{0}
$$

where $P_{0}$ is a standard pressure at the Earth's surface.

In this paper, we will consider an analytical solution of the baroclinic instability problem using an eigenvalue approach for a two-layer model. The model vertical structure is shown in Figure 1. For this model the boundary condition (10) gives $\omega_{2}=\omega_{4}=0$. Applying quasi-geostrophic vorticity equation (8) to the 750 and $250-\mathrm{hPa}$ surfaces and approximating the derivative $\partial \omega / \partial p$ by finite differences one can obtain the resulting vorticity equations at levels 1 and 3 :

$$
\begin{aligned}
& \frac{\partial}{\partial t} \nabla^{2} \psi_{1}+\mathbf{u}_{1} \cdot \nabla\left(\nabla^{2} \psi_{1}\right)+\beta \frac{\partial \psi}{\partial x}=\frac{f_{0}}{\Delta p} \omega_{2}, \\
& \frac{\partial}{\partial t} \nabla^{2} \psi_{3}+\mathbf{u}_{3} \cdot \nabla\left(\nabla^{2} \psi_{3}\right)+\beta \frac{\partial \psi}{\partial x}=-\frac{f_{0}}{\Delta p} \omega_{2},
\end{aligned}
$$

where $\mathbf{u}=u \hat{i}+v \hat{j}$ is the horizontal velocity and $\Delta p=500 \mathrm{hPa}$. The thermodynamic energy equation (10) is applied at level 2 :

$$
\frac{\partial}{\partial t}\left(\psi_{1}-\psi_{3}\right)+\mathbf{u}_{2} \cdot \nabla\left(\psi_{1}-\psi_{3}\right)-\frac{\sigma \Delta p}{f_{0}} \omega_{2}=0
$$

Thus, we have a system of three equations (11)-(13) in the three variables $\omega_{2}, \psi_{1}$, and $\psi_{3}$. To study the instability of the basic zonal flow with respect to infinitesimal perturbations, these equations are linearized around the basic state (6). Let us assume that

$$
\begin{aligned}
& \psi_{1}=-u_{1} y+\psi_{1}^{\prime}(x, t), \\
& \psi_{3}=-u_{3} y+\psi_{3}^{\prime}(x, t), \\
& \omega_{2}=\omega_{2}^{\prime}(x, t) .
\end{aligned}
$$

Substituting (14) into (11)-(13), defining

$$
\begin{aligned}
& u_{m}=\frac{1}{2}\left(u_{1}+u_{3}\right), \\
& u_{T}=\frac{1}{2}\left(u_{1}-u_{3}\right), \\
& \psi_{m}=\frac{1}{2}\left(\psi_{1}^{\prime}+\psi_{3}^{\prime}\right), \\
& \psi_{T}=\frac{1}{2}\left(\psi_{1}^{\prime}-\psi_{3}^{\prime}\right),
\end{aligned}
$$

and eliminating the variable $\omega_{2}^{\prime}$ yield the following perturbation equations:

$$
\begin{aligned}
& \left(\frac{\partial}{\partial t}+u_{m} \frac{\partial}{\partial x}\right) \frac{\partial^{2} \psi_{m}}{\partial x^{2}}+\beta \frac{\partial \psi_{m}}{\partial x}+u_{T} \frac{\partial}{\partial x}\left(\frac{\partial^{2} \psi_{T}}{\partial x^{2}}\right)=0, \\
& \left(\frac{\partial}{\partial t}+u_{m} \frac{\partial}{\partial x}\right)\left(\frac{\partial^{2} \psi_{T}}{\partial x^{2}}-2 \lambda^{2} \psi_{T}\right)+\beta \frac{\partial \psi_{T}}{\partial x} \\
& +u_{T} \frac{\partial}{\partial x}\left(\frac{\partial^{2} \psi_{T}}{\partial x^{2}}+2 \lambda^{2} \psi_{T}\right)=0,
\end{aligned}
$$

where $\lambda^{2}=f_{0}^{2} /\left[\sigma(\Delta p)^{2}\right]$. We will seek normal mode solutions of the following form:

$$
\begin{aligned}
& \psi_{m}(x, t)=\Psi_{m} e^{i k(x-c t)}, \\
& \psi_{T}(x, t)=\Psi_{T} e^{i k(x-c t)},
\end{aligned}
$$

where $\Psi_{m}$ and $\Psi_{T}$ are the amplitude of perturbations, $k$ is a wavenumber, and $c$ is a complex phase velocity. By substituting (17) into (16), after some algebraic manipulation we can obtain the following equation for the phase speed of baroclinic waves:

$$
c=u_{m}-\frac{\beta}{k^{2}} \frac{k^{2}+\lambda^{2}}{k^{2}+2 \lambda^{2}} \pm \sqrt{\delta}
$$

where

$$
\delta=\frac{\beta^{2} \lambda^{4}}{k^{4}\left(k^{2}+2 \lambda^{2}\right)^{2}}+u_{T}^{2} \frac{k^{2}-2 \lambda^{2}}{k^{2}+2 \lambda^{2}} .
$$

Perturbations will grow exponentially if $c$ has an imaginary part $c_{i}$. This will occur if $\delta<0$. 


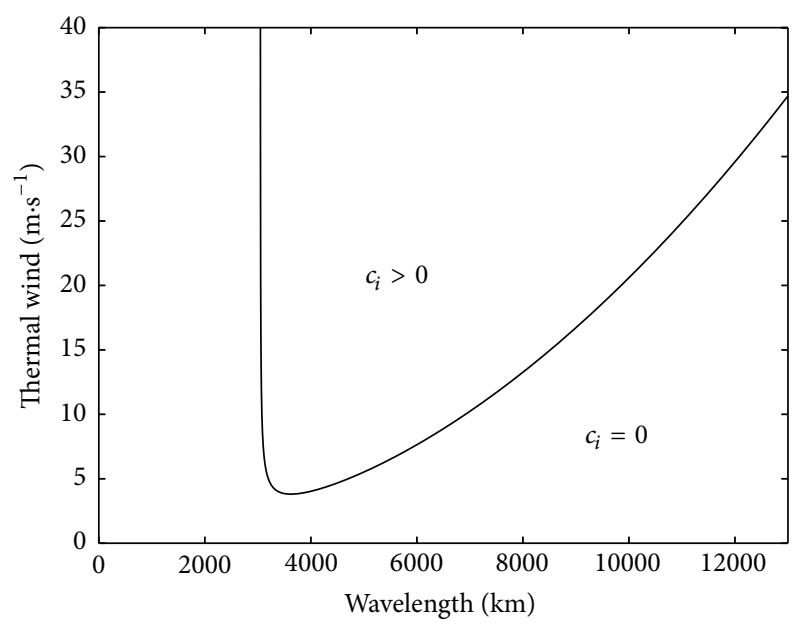

FIGURE 2: Instability diagram displaying wavelength regions of stable and unstable waves.

Then the increment of growing mode with a wavenumber $k$ is given by the following expression:

$$
\chi_{k} \equiv k c_{i}=\frac{\sqrt{\left|\beta^{2} \lambda^{4}+u_{T}^{2} k^{4}\left(k^{4}-4 \lambda^{4}\right)\right|}}{k\left(k^{2}+2 \lambda^{2}\right)} .
$$

This equation shows that the growth rate of unstable perturbations depends on the wind shear $u_{T}$ associated with the pole-equator temperature gradient and the variable $\lambda$, which is a function of the static stability $\sigma$. Thus, parameters $u_{T}$ and $\sigma$ can be considered as feasible controls.

\section{Sensitivity Analysis of Baroclinic Instability in the Context of Atmospheric Dynamics Control}

First, let us highlight the most important properties of the model described in Section 3. By setting the discriminant (19) equal to zero, the so-called marginal stability curve (neutral curve) that separates the stable region from the unstable region can be plotted as function of the mean thermal wind, $u_{T}$, and perturbation wavenumber, $k$ (see Figure 2 ):

$$
u_{T}^{2}=\frac{\beta^{2} \lambda^{4}}{k^{4}\left(4 \lambda^{4}-k^{4}\right)} .
$$

Figure 2 shows that there are two stable regimes, one for short waves and another one for long waves. Thus, two-layer model has a shortwave cut-off $\left(L_{S C}\right)$ and longwave cut-off $\left(L_{\mathrm{LC}}\right)$ : any particular wave is unstable if its length $L_{x}$ satisfies the following double inequalities $L_{\mathrm{SC}}<L_{x}<L_{\mathrm{LC}}$. The range of unstable waves depends on static stability and thermal wind and can be found from (21) by the following way:

$$
k^{4}=2 \lambda^{4} \pm\left(4 \lambda^{8}-\frac{\beta^{2} \lambda^{4}}{u_{T}^{2}}\right)^{1 / 2} .
$$

If the thermal wind is less than the minimum value $u_{T}^{\min }$ on the stability curve, then all waves are stable. The minimum thermal wind required for the development of instability can be estimated by differentiating (21) with respect to $k:\left|u_{T}^{\min }\right|>$ $\beta /\left(2 \lambda^{2}\right)$. If the thermal wind exceeds the value of $u_{T}^{\min }$, then both stable and unstable waves can exist.

The dispersion diagram (Figure 3 ) shows that baroclinic waves of different wavelengths travel in space at different velocities. In Figure 3 two values of the phase velocity correspond to stable waves and one value to unstable waves. Short and long waves both stable and unstable travel eastward. Only very long waves $\left(L_{x}>6000 \mathrm{~km}\right)$ can propagate westward; however, these waves are outside of our interest. Phase speed of quasi-barotropic stable Rossby waves is also shown in Figure 3 for comparison. These results are obtained for $f_{0}=$ $1.03 \mathrm{~s}^{-1}$ that matches the latitude $\varphi_{0}=45^{\circ} \mathrm{N}, \beta=1.63 \times$ $10^{-11} \mathrm{~m}^{-1} \mathrm{~s}^{-1}, \sigma=2 \times 10^{-6} \mathrm{~m}^{2} \mathrm{~Pa}^{-2} \mathrm{~s}^{-2}, u_{T}=7.5 \mathrm{~m} \mathrm{~s}^{-1}$, and $u_{m}=15 \mathrm{~m} \mathrm{~s}^{-1}$ [23].

In the two-layer model, the static stability parameter $\sigma$ and the vertical wind shear $u_{T}$ control the development of baroclinic instability. To estimate the influence of control actions of the development of baroclinic instability the sensitivity coefficients $S_{\sigma}$ and $S_{u_{T}}$ are employed. Analytical expressions for $S_{\sigma}$ and $S_{u_{T}}$ are obtained by differentiating (20) with respect to $\sigma$ and $u_{T}$ :

$$
\begin{aligned}
S_{\sigma} & \equiv \frac{\partial \chi_{k}}{\partial \sigma}=-\frac{k \lambda^{2}}{\sigma\left(k^{2}+2 \lambda^{2}\right)^{2}} \frac{2 u_{T}^{2} k^{4}\left(k^{4}+2 \lambda^{2}\right)-\beta^{2} \lambda^{2}}{u_{T}^{2} k^{4}\left(k^{4}-4 \lambda^{4}\right)+\beta^{2} \lambda^{4}} \\
& \cdot \sqrt{u_{T}^{2} k^{4}\left(k^{4}-4 \lambda^{4}\right)+\beta^{2} \lambda^{4}}, \\
S_{u_{T}} & \equiv \frac{\partial \chi_{k}}{\partial u_{T}}=-u_{T} k^{3}\left(k^{2}-2 \lambda^{2}\right) \\
& \cdot \frac{\sqrt{\left|\beta^{2} \lambda^{4}+u_{T}^{2} k^{4}\left(k^{4}-4 \lambda^{4}\right)\right|}}{\beta^{2} \lambda^{4}+u_{T}^{2} k^{4}\left(k^{4}-4 \lambda^{4}\right)} .
\end{aligned}
$$

Sensitivity coefficients $S_{\sigma}$ and $S_{u_{T}}$ should be estimated in the vicinity of some reference values of the static stability parameter $\sigma^{*}$ and thermal wind $u_{T}^{*}$, respectively, which depend on the chosen weather and climate conditions.

Sensitivity coefficients $S_{\sigma}$ calculated for different basic values of the static stability parameter $\sigma^{*}$ provide important information regarding the impact of $\sigma^{*}$ on the growth rate of unstable waves. As shown in Figure 4, the absolute values of sensitivity coefficients $S_{\sigma}$ exponentially increase with decreasing wavelength for a specified value of $\sigma^{*}$. As an example let us consider two waves $(A$ and $B$ ) of different wavelengths, $L_{x}^{(A)} \approx 3,000$ and $L_{x}^{(B)} \approx 5,000 \mathrm{~km}$, respectively, for the case of $\sigma^{*}=2 \times 10^{-6} \mathrm{~m}^{2} \cdot \mathrm{Pa}^{-2} \cdot \mathrm{s}^{-2}$ and $u_{T}^{*}=7.5 \mathrm{~m} \cdot \mathrm{s}^{-1}$. The sensitivity of wave $A$ with respect to the static stability parameter $S_{\sigma}^{(A)} \approx-16$ is about 12 times the sensitivity $S_{\sigma}^{(B)} \approx-1.35$ of wave $B$ in absolute value. Thus, short baroclinic unstable waves possess a high sensitivity to the atmospheric static stability: the smaller the wavelength, the higher the sensitivity. In contrast, long unstable waves are more sensitive to the vertical wind shear but not to the static stability (Figure 5). The sensitivity of long unstable wave $B$ 


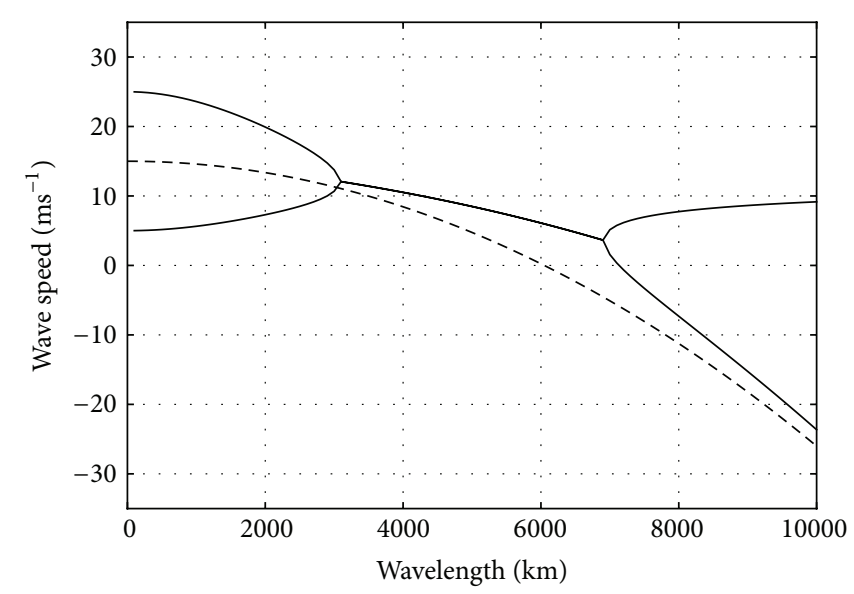

Figure 3: Phase velocity as a function of wavelength for baroclinic waves (solid line) and for Rossby waves (dashed line) for the case of $\sigma=2 \times 10^{-6} \mathrm{~m}^{2} \cdot \mathrm{Pa}^{-2} \cdot \mathrm{s}^{-2}, u_{T}=7.5 \mathrm{~m} \cdot \mathrm{s}^{-1}$, and $u_{m}=15 \mathrm{~m} \cdot \mathrm{s}^{-1}$.

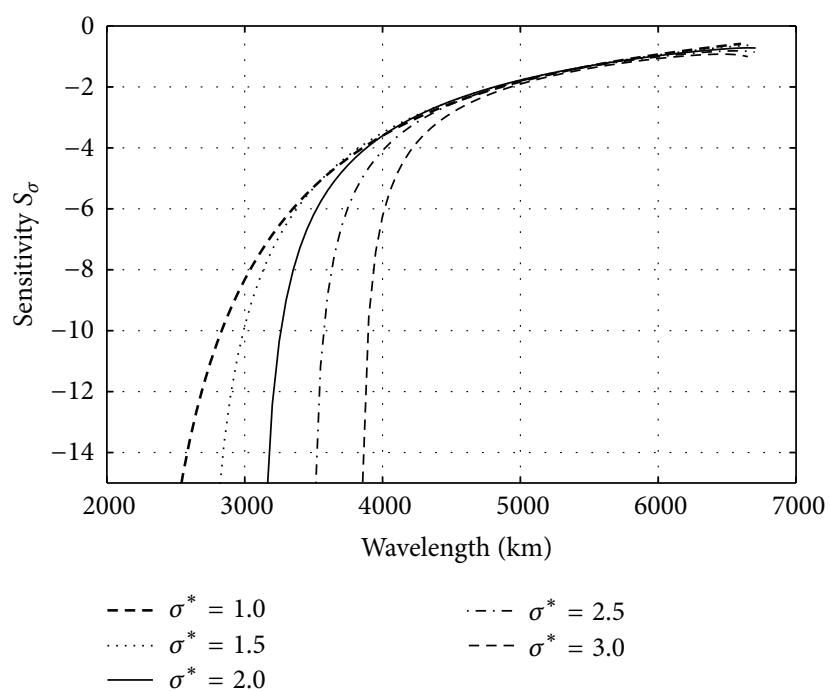

FIGURE 4: Sensitivity coefficients $S_{\sigma}$ versus the wavelength for different reference values of static stability parameter $\sigma^{*} \times 10^{6} \mathrm{~m}^{2} \cdot \mathrm{Pa}^{-2} \cdot \mathrm{s}^{-2}$ for the case of $u_{T}=7.5 \mathrm{~m} \cdot \mathrm{s}^{-1}$.

with respect to the wind shear $S_{u_{T}}^{(B)} \approx 0.11$ is about 2 times the sensitivity $S_{u_{T}}^{(A)} \approx 0.05$ of short unstable wave $A$.

If $\delta \sigma$ and $\delta u$ represent the control actions such that $\delta \sigma \ll$ $\sigma^{*}$ and $\delta u \ll u_{T}^{*}$, the unstable wave growth rate changes $\delta \chi_{k}$ induced by $\delta \sigma$ and $\delta u$ are estimated to a first-order accuracy in the following way:

$$
\begin{aligned}
& \delta \chi_{k}(\delta \sigma) \equiv \chi_{k}\left(\sigma^{*}+\delta \sigma\right)-\chi_{k}\left(\sigma^{*}\right) \approx \delta \sigma \times\left. S_{\sigma}\right|_{\sigma=\sigma *}, \\
& \delta \chi_{k}(\delta u) \equiv \chi_{k}\left(u_{T}^{*}+\delta u\right)-\chi_{k}\left(u_{T}^{*}\right) \approx \delta u \times\left. S_{u_{T}}\right|_{u_{T}=u_{T}^{*}}
\end{aligned}
$$

Suppose the reference value of the static stability $\sigma^{*}$ is equal to $2 \times 10^{-6} \mathrm{~m}^{2} \cdot \mathrm{Pa}^{-2} \cdot \mathrm{s}^{-1}$ [23], which is a typical midlatitude tropospheric value of the static stability parameter. Let us use (24) to estimate the impact of control $\delta \sigma$ on the growth rates of unstable perturbations, assuming that $\delta \sigma$ is minus 5 percentage points of $\sigma^{*}$. Note that this decrease in the static stability parameter corresponds to a $0.2 \mathrm{~K} \cdot \mathrm{km}^{-1}$ increase in the lapse rate $\gamma$, while the standard tropospheric value of $\gamma$ is $6.5 \mathrm{~K} \cdot \mathrm{km}^{-1}$ [23]. The mentioned above lapse rate change may be achieved in various ways, for example, by changing the surface albedo. Table 1 illustrates changes in the growth rates of unstable waves caused by control $\delta \sigma$. The most important result is that the short unstable wave of wavelength $L_{x} \approx L_{x}^{(A)}$ demonstrates the phenomenal change in the growth rate $\delta \chi$ of nearly 87 per cent compared to the value that corresponds to the unperturbed static stability parameter $\sigma^{*}$. Its growth rate reaches the value of $0.28 \mathrm{day}^{-1}$. The influence of $\delta \sigma$ on the growth rates of unstable perturbations tends to decrease rapidly with increasing wavelength. So, small perturbations in the static stability lead to the tangible changes to the growth rates of short baroclinic unstable waves $\left(L_{x} \sim L_{x}^{(A)}\right)$.

The development of baroclinic instability can be partially suppressed if the static stability is increased due to control actions. The suppression degree, in accordance with Figure 4, 


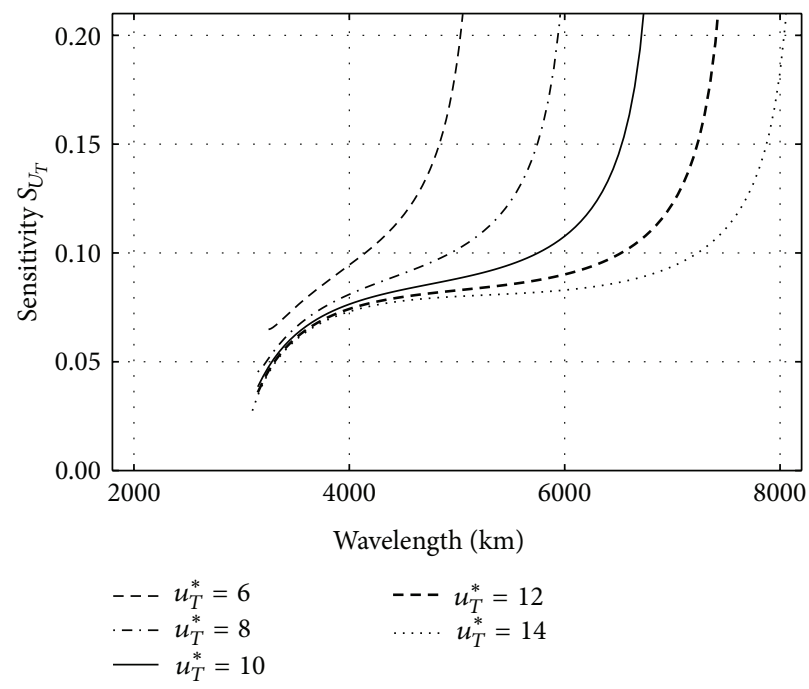

FIGURE 5: Sensitivity coefficients $S_{u_{T}}$ versus the wavelength for different reference values of thermal wind $u_{T}^{*}$ for the case of $\sigma=2 \times$ $10^{-6} \mathrm{~m}^{2} \cdot \mathrm{Pa}^{-2} \cdot \mathrm{s}^{-2}$.

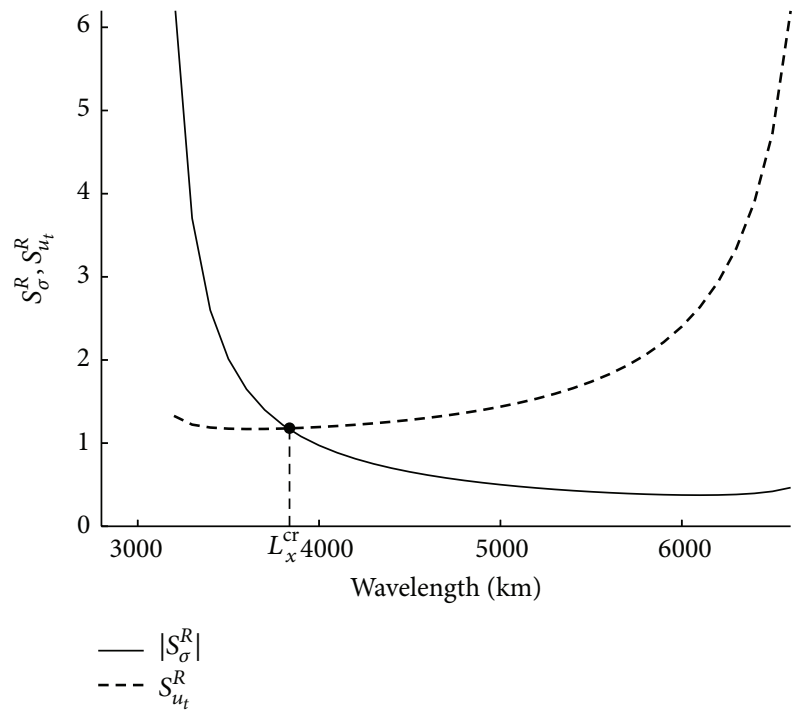

FIGURE 6: Relative sensitivity coefficients $S_{\sigma}^{R}$ and $S_{u_{T}}^{R}$ versus the wavelength $L_{x}$ for the case of $\sigma=2 \times 10^{-6} \mathrm{~m}^{2} \cdot \mathrm{Pa}^{-2} \cdot \mathrm{s}^{-2}$ and $u_{T}=$ $7.5 \mathrm{~m} \cdot \mathrm{s}^{-1}$.

is also dependent on the wavelength. For example, if the control $\delta \sigma$ is 5 percentage points of $\sigma^{*}$, then the amplitude of the short unstable wave $A$ does not grow.

Let us examine now the influence of variations in the thermal wind on changes in the growth rates of baroclinic unstable waves using (25). In calculations, the reference value of vertical wind share $u_{T}^{*}$ was set equal to $7.5 \mathrm{~m} \cdot \mathrm{s}^{-1}$, which corresponds to the vertical gradient of wind velocity equal to $0.03 \mathrm{~m} \cdot \mathrm{s}^{-1} \cdot \mathrm{hPa}^{-1}$ [23]. According to the thermal wind balance, this wind shear is generated by the meridional temperature gradient equal to $5.2^{\circ} \mathrm{K}$ per $1000 \mathrm{~km}$. Let the control $\delta u_{T}$ be 5 percentage points of the reference value $u_{T}^{*}$.
TABLE 1: Changes in the growth rates of unstable waves for the case of $\delta \sigma=-0,05 \times \sigma^{*}, \sigma^{*}=2 \times 10^{-6} \mathrm{~m}^{2} \cdot \mathrm{Pa}^{-2} \cdot \mathrm{s}^{-1}$, and $u_{T}^{*}=7.5 \mathrm{~m} \cdot \mathrm{s}^{-1}$.

\begin{tabular}{lccccc}
\hline$L_{x} \mathrm{~km}$ & 5000 & 4000 & 3500 & 3200 & 3150 \\
$\chi$ day $^{-1}$ & 0.37 & 0.44 & 0.37 & 0.21 & 0.15 \\
$S_{\sigma}$ & -1.34 & -2.77 & -4.85 & -10.60 & -15.31 \\
$\delta \chi$ day $^{-1}$ & 0.01 & 0.02 & 0.04 & 0.09 & 0.13 \\
$(\delta \chi / \chi) \times 100 \%$ & 2.7 & 4.5 & 10.8 & 42.9 & 86.7 \\
\hline
\end{tabular}

TABLE 2: Changes in the growth rates of unstable waves for the case of $\delta u_{T}=0.05 \times u_{T}^{*}, \sigma^{*}=2 \times 10^{-6} \mathrm{~m}^{2} \cdot \mathrm{Pa}^{-2} \cdot \mathrm{s}^{-1}$, and $u_{T}^{*}=7.5 \mathrm{~m} \cdot \mathrm{s}^{-1}$.

\begin{tabular}{lccccc}
\hline$L_{x} \mathrm{~km}$ & 5000 & 4000 & 3500 & 3200 & 3150 \\
$S_{u_{T}}$ & 0.11 & 0.08 & 0.07 & 0.05 & 0.05 \\
$\delta \chi$ day $^{-1}$ & 0.04 & 0.03 & 0.03 & 0.03 & 0.02 \\
$(\delta \chi / \chi) \times 100 \%$ & 10.8 & 6.8 & 8.1 & 9.5 & 12.3 \\
\hline
\end{tabular}

Table 2 shows changes in the growth rates of unstable waves caused by control $\delta u_{T}$. From this table, it follows that the relative change in growth rates of both short and long waves is about 10 percent with respect to that of the unperturbed reference value of the wind shear.

The results presented in Tables 1 and 2 can be summarized as follows: changing the vertical stratification of the atmosphere is the most appropriate mechanism for controlling the baroclinic instability.

Since control parameters $\delta \sigma$ and $\delta u_{T}$ have different dimensions and different equivalence classes, in order to compare their relative role in changing $\delta \chi$, we can use relative (normalized) sensitivity coefficients:

$$
\begin{aligned}
S_{\sigma}^{R} & =S_{\sigma} \frac{\sigma}{\chi_{k}}, \\
S_{u_{T}}^{R} & =S_{u_{T}} \frac{u_{T}}{\chi_{k}} .
\end{aligned}
$$


The analysis of the relative sensitivity coefficients leads to an important conclusion. There is a critical value of the wavelength $L_{x}^{\mathrm{cr}}$ that divides the spectrum of unstable waves into two parts (Figure 6). The development of baroclinic instability is mainly affected by the atmospheric static stability for the case of short-wavelengths $\left(L_{x}<L_{x}^{\mathrm{cr}}\right)$. However, if $L_{x}>L_{x}^{\mathrm{cr}}$ then the prevailing role in the development of baroclinic instability plays the vertical wind shear, that is, the meridional temperature gradient. For example, if $\sigma^{*}=$ $2 \times 10^{-6} \mathrm{~m}^{2} \cdot \mathrm{Pa}^{-2} \cdot \mathrm{s}^{-1}$ and $u_{T}=7.5 \mathrm{~m} \cdot \mathrm{s}^{-1}$, then $L_{x}^{\mathrm{cr}} \approx 3800 \mathrm{~km}$.

\section{Concluding Remarks}

Geoengineering has appeared as a potential option to reduce the impacts of climate change. So far, however, the effectiveness of geoengineering methods is examined outside of the scope of optimal control theory, and geoengineering itself is considered as an intentional action to influence natural climate processes. Geophysical cybernetics provides a conceptual and unified theoretical framework for developing and synthesizing the optimal control systems for natural environmental phenomena and processes. The application of geophysical cybernetics requires a suitable mathematical model of the ECS. In mathematical climate models, control actions that manipulate the weather and climate can be expressed via variations in the model parameters chosen as control variables. It is very important that control variables should be physically feasible. The use of sensitivity theory in dynamical systems allows one to determine control variables that satisfy this requirement.

In this paper, bearing in mind the control problem for large-scale atmospheric dynamics, we considered the atmospheric baroclinic instability as the controlled object. Within the framework of two-layer atmospheric model used in this study there are two fundamental atmospheric parameters that govern the development of baroclinic instability, namely, the static stability and the vertical wind shear induced by the meridional temperature gradient. The influence of small variations in these two parameters on the development of baroclinic instability has been studied. Analytical expressions were derived for absolute and relative sensitivity coefficients that allow one to estimate the absolute and relative contribution of variations in the static stability and vertical wind shear to changes in the growth rates of unstable baroclinic waves. It was shown that changing the vertical stratification of the atmosphere (i.e., changing the static stability) is the most appropriate method for controlling the baroclinic instability. The influence of meridional temperature gradient on the growth rate of unstable waves is less significant. Therefore, the vertical wind shear can hardly be regarded as a control variable.

Let us emphasize that climate manipulation is a multidisciplinary research area that requires consideration not only of the mathematical aspects but also of the physical, chemical, technical, ethical, and legal aspects and limitations. The interest in manipulation of the climate and weather will likely continue to grow, which requires the development of theoretical foundation for the optimal control of the ECS. The approach outlined in this paper is expected to be applied for the study of sensitivity of climate and atmospheric models in order to estimate the hypothetical possibility of weather and climate optimal control.

\section{Competing Interests}

The authors declare that they have no competing interests.

\section{References}

[1] A. S. Dennis, Changing of Weather by Cloud Seeding, Academic Press, New York, NY, USA, 1980.

[2] M. Ćurić, D. Janc, and V. Vučković, "Cloud seeding impact on precipitation as revealed by cloud-resolving mesoscale model," Meteorology and Atmospheric Physics, vol. 95, no. 3-4, pp. 179193, 2007.

[3] D. L. Mitchell and W. Finnegan, "Modification of cirrus clouds to reduce global warming," Environmental Research Letters, vol. 4, no. 4, Article ID 045102, 2009.

[4] X. Guo, D. Fu, X. Li et al., "Advances in cloud physics and weather modification in China," Advances in Atmospheric Sciences, vol. 32, no. 2, pp. 230-249, 2015.

[5] I. Colbeck, "The development of fog intensive dispersal operation," in Aerosol Science and Technology: Hystory and Reviews, D. S. Ensor, Ed., pp. 367-375, RTI Press, Research Triangle Park, NC, USA, 2011.

[6] R. N. Hoffman, "Controlling the global weather," Bulletin of the American Meteorological Society, vol. 83, no. 2, pp. 241-248, 2002.

[7] M. Garstang, R. Bruintjes, R. Serafin et al., "Weather modification: finding common ground," Bulletin of the American Meteorological Society, vol. 86, no. 5, pp. 647-655, 2005.

[8] J. R. Fleming, Fixing the Sky: The Checkered History of Weather and Climate Control, Columbia University Press, New York, NY, USA, 2010.

[9] Geoengineering the Climate: Science, Governance and Uncertainty, The Royal Society, 2009.

[10] T. F. Stocker, D. Qin, G.-K. Plattner et al., Eds., Climate Change 2013: The Physical Science Basis. Contribution of Working Group I to the Fifth Assessment Report of the Intergovernmental Panel on Climate Change, Cambridge University Press, Cambridge, UK, 2013.

[11] M. C. MacCracken, "On the possible use of geoengineering to moderate specific climate change impacts," Environmental Research Letters, vol. 4, no. 4, Article ID 045107, pp. 1-14, 2009.

[12] T. Ming, R. De Richter, W. Liu, and S. Caillol, "Fighting global warming by climate engineering: is the Earth radiation management and the solar radiation management any option for fighting climate change," Renewable and Sustainable Energy Reviews, vol. 31, pp. 792-834, 2014.

[13] R. M. Yusupov, Theoretical Bases of Control of Geophysical Processes, Ministry of Higher Education of the USSR, Moscow, Russia, 1979.

[14] D. V. Gaskarov, V. B. Kisselev, S. A. Soldatenko, V. I. Strogonov, and R. M. Yusupov, An Introduction to Geophysical Cybernetics and Environmental Monitoring, St. Petersburg State University, St. Petersburg, Russia, 1998.

[15] S. Soldatenko and R. Yusupov, "On the possible use of geophysical cybernetics in climate manipulation (geoengineering) and 
weather modification," WSEAS Transactions on Environment and Development, vol. 11, pp. 116-125, 2015.

[16] S. Soldatenko and R. Yusupov, "An optimal control problem formulation for the atmospheric large-scale wave dynamics," Applied Mathematical Sciences, vol. 9, no. 17-20, pp. 875-884, 2015.

[17] H. A. Dijkstra, Nonlinear Climate Dynamics, Cambridge University Press, New York, NY, USA, 2013.

[18] E. Rosenwasser and R. Yusupov, Sensitivity of Automatic Control Systems, CRC Press, Boca Raton, Fla, USA, 2000.

[19] D. G. Cacuci, Sensitivity and Uncertainty Analysis, Volume I: Theory, CRC, Boca Raton, Fla, USA, 2003.

[20] J. Pedlosky, Geophysical Fluid Dynamics, Springer, New York, NY, USA, 1987.

[21] M. L. Salby, Fundamental of Atmospheric Physics, Academic Press, San Diego, Calif, USA, 1996.

[22] E. N. Lorenz, "Deterministic nonperiodic flow," Journal of the Atmospheric Sciences, vol. 20, pp. 130-140, 1963.

[23] J. R. Holton, An Introduction to Dynamic Meteorology, Elsevier, London, UK, 4th edition, 2004. 


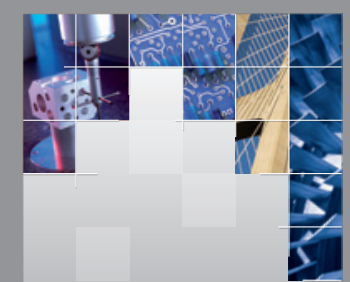

\section{Enfincering}
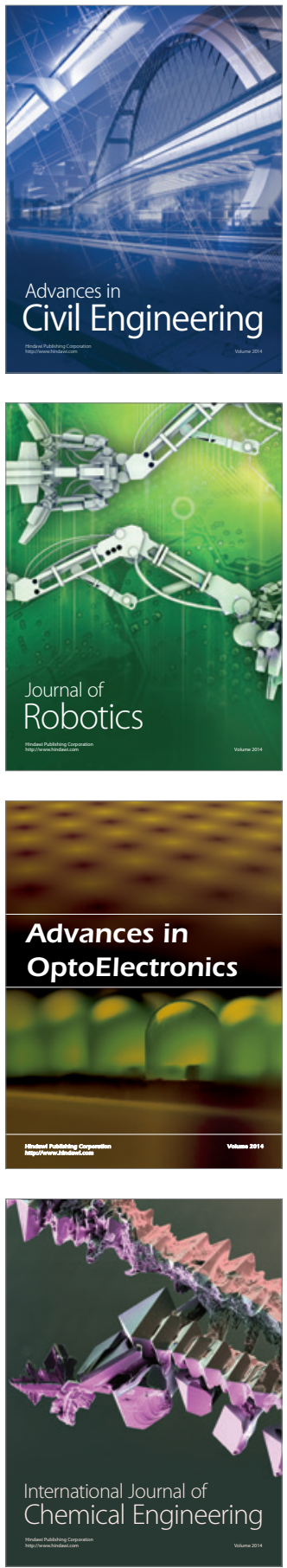

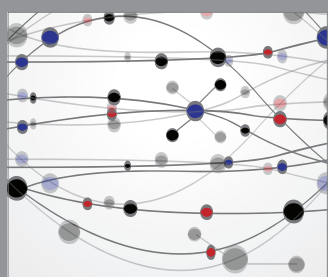

The Scientific World Journal

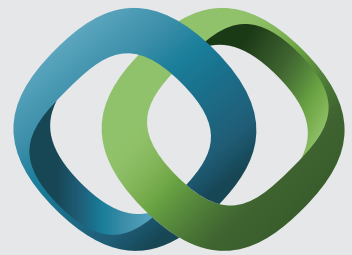

\section{Hindawi}

Submit your manuscripts at

http://www.hindawi.com
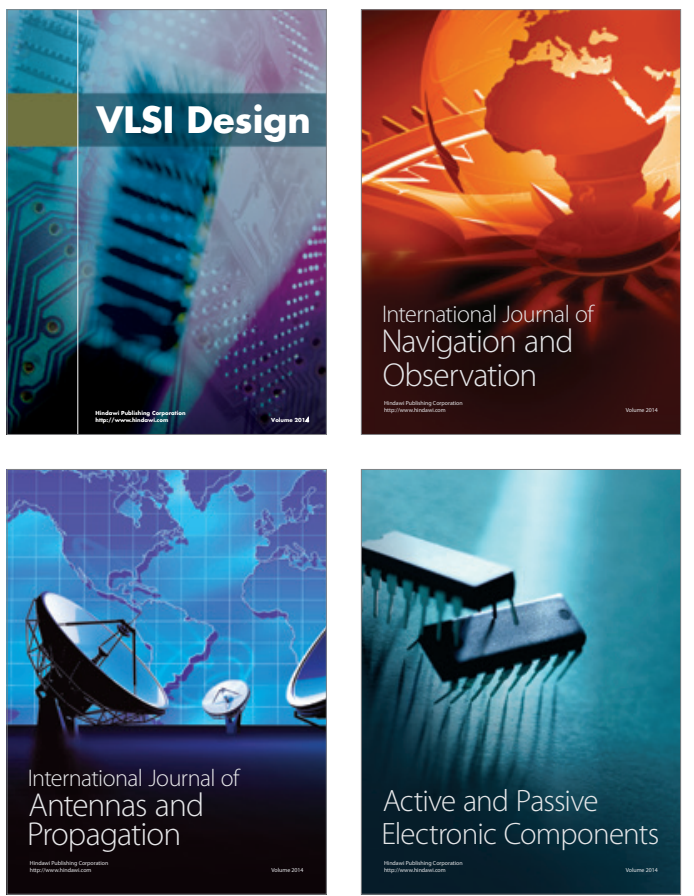
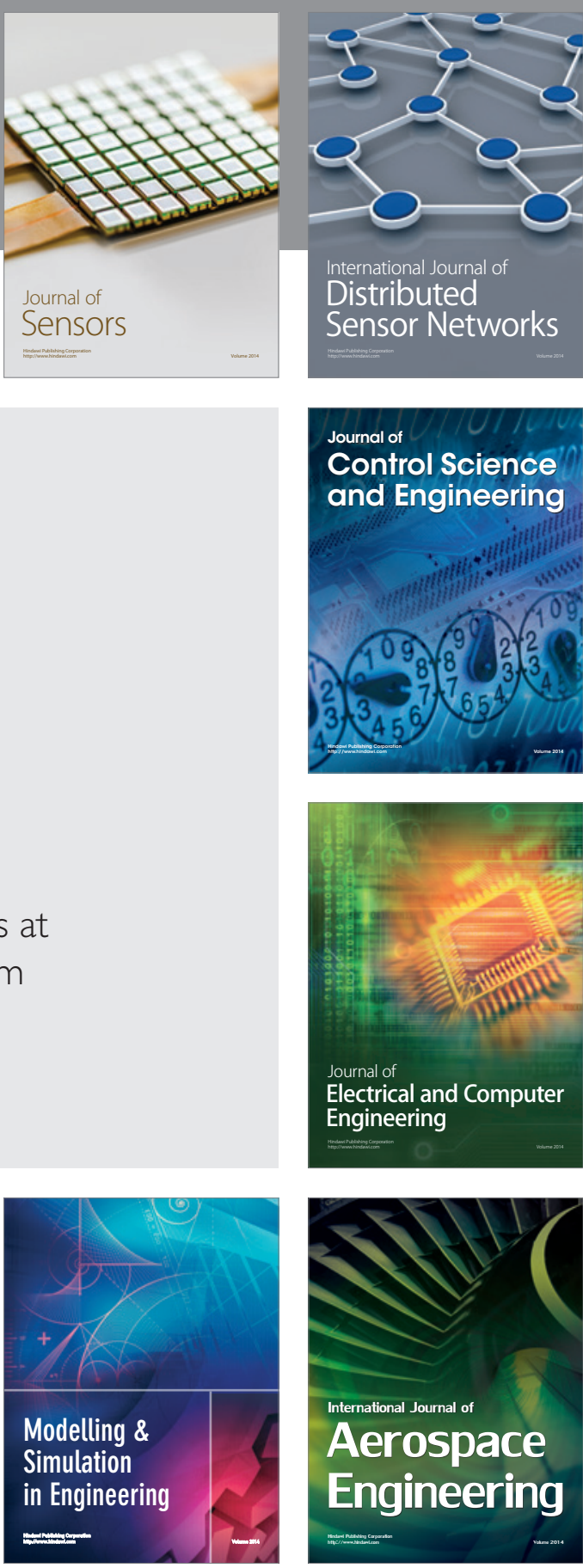

International Journal of

Distributed

Sensor Networks

Journal of

Control Science

and Engineering
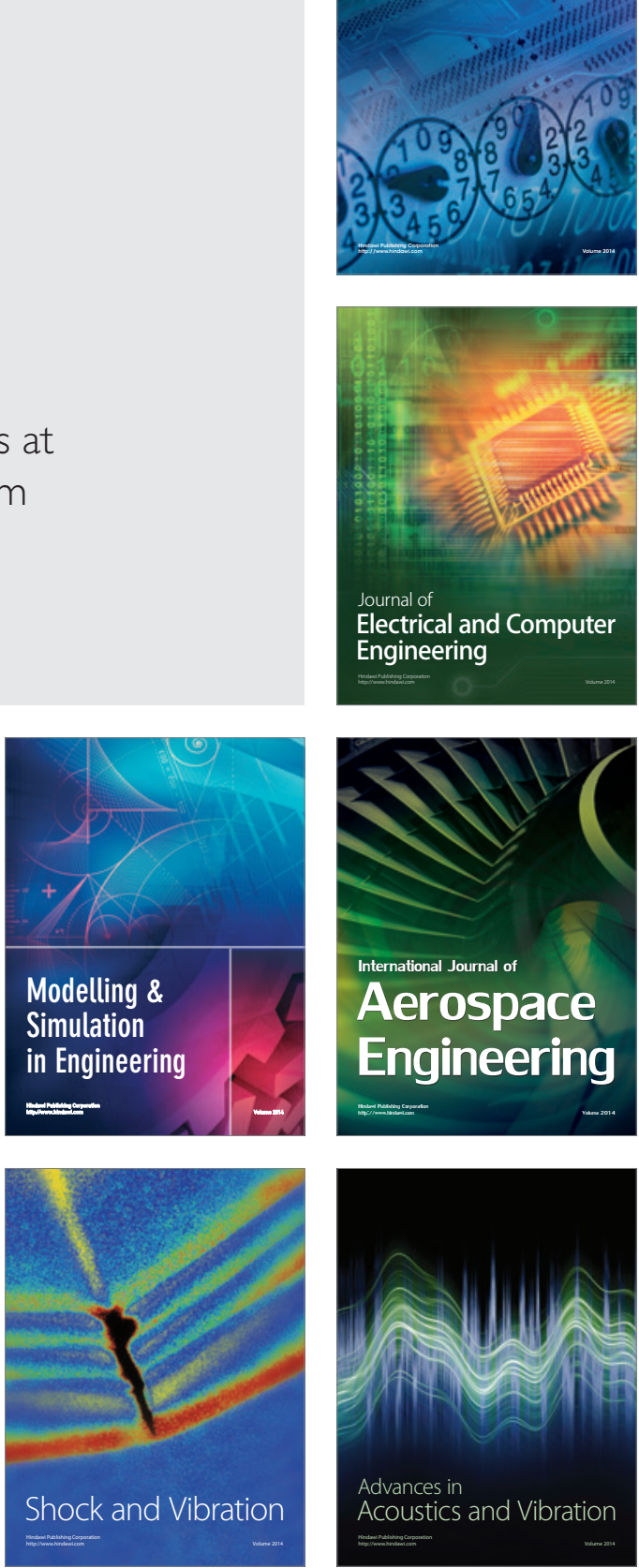